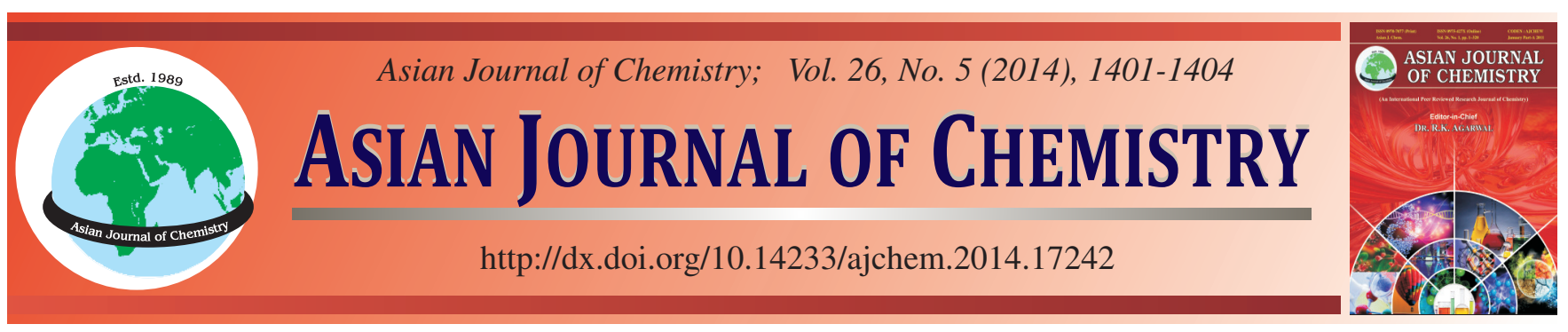

\title{
Morphological Control of Mesoporous Silica Nanoparticles and Their Application for Foam Stability $\dagger$
}

\begin{abstract}
Suk Bon Yoon ${ }^{1,2}$, In-Ho Yoon ${ }^{1}$, Chorong Kim ${ }^{1}$, Chong-Hun Jung ${ }^{1}$, Seonbyeong Kim ${ }^{1}$, Wang-Kyu Chol ${ }^{1, *}$ and Jei-Kwon Moon ${ }^{1}$
${ }^{1}$ Decontamination and Decommissioning Research Division, Korea Atomic Energy Research Institute, Daejeon 305-353, Republic of Korea ${ }^{2}$ Central Research Institute, Korea Hydro \& Nuclear Power Co., Ltd. (KHNP-CRI), Daejeon 305-343, Republic of Korea
\end{abstract}

*Corresponding author: Fax: +82 42 8682328; E-mail: nwkchoi@ kaeri.re.kr

Published online: 1 March 2014;

\begin{abstract}
Mesoporous silica nanoparticles with various sizes and shapes were synthesized by varying the amount of the ammonia solution as a basic catalyst and cetyltrimethylammonium bromide $(\mathrm{CTABr})$ as a cationic surfactant. It was found that the particle size and shape were strongly dependent on the amount of the ammonia solution and cetyltrimethylammonium bromide. While the mesoporous silicas exhibited the small sizes and irregular shapes when using a low concentration of the ammonia solution, the rod-like shapes with well-ordered pore structures as a MCM-41 were synthesized under a strong basic condition. Resulting materials showed high specific surface areas (813$\left.919 \mathrm{~m}^{2} / \mathrm{g}\right)$, large pore volumes $\left(0.60-1.66 \mathrm{~cm}^{3} / \mathrm{g}\right)$ and uniform pore sizes $(1.87-2.36 \mathrm{~nm})$. The mesoporous silica nanoparticles with different sizes were used to investigate the effect of the nanoparticles on the foam stability. The results showed that both foam volume and liquid volume in the foam were enhanced when using a smaller size and lower density of the mesoporous silica nanopartcles.
\end{abstract}

Keywords: Morphology, Mesoporous silica, MCM-41, Nanoparticle, Foam stability.

\section{INTRODUCTION}

A mesoporous silica is one of the important inorganic nanoporous materials because it has various applications in a wide variety of the research fields such as separation and purification, catalysis, adsorption and advanced nanomaterials owing to their ordered and uniform pore structures and high specific surface areas ${ }^{1-4}$. The synthesis of mesoporous silicas has been mainly accomplished through a self-assembly between surfactant molecules and inorganic species under various $\mathrm{pH}$ conditions $\mathrm{s}^{5-7}$. The pore sizes of mesoporous silicas found in literatures can be easily controlled by changing the alkyl-chain length of the surfactant. The control of the particle size and shape is necessary for various practical applications. The synthesis of spherical mesoporous silica nanoparticles with hexagonal and cubic mesostructures previously using modified Stöber method was reported $^{8,9}$. But the resulting particles were polydisperse and nonspherical in some $\operatorname{cases}^{10}$. In our previous study, the coreshell nanoparticles with a silica core and mesoporous shell under basic conditions were synthesized through a combination of a self-assembly of a cationic surfactant and a sol-gel reaction of silica precursor on the surface of the silica nanoparticles ${ }^{11}$.
Recently, a few research for the preparation of the silica based-chemical gel ${ }^{12,13}$ and decontamination foam containing solid nanoparticles ${ }^{14}$ in an effort to reduce the amount of radioactive waste as well as enhance the decontamination efficiency during the decontamination process of nuclear facilities contaminated with radionuclides have been reported. However, most of the research for the foam has focused on industrial fields such as food, cosmetics, home and personal care products, etc. and the study for the decontamination foam to enhance the decontamination efficiency and reduce the chemical reagents and secondary wastes produced during the decontamination process is not so common.

In this work, we report the synthesis of the mesoporous silica nanoparticles with various sizes and shapes by controlling the amount of the ammonia solution and surfactant under a TEOS/CTABr/ $/ \mathrm{NH}_{4} \mathrm{OH} / \mathrm{H}_{2} \mathrm{O}$ system and their applications for the foam stability. As the amount of the ammonia solution increases, resulting materials exhibited various sizes and shapes such as spherical, rod-like and hexagonal. In a study on the foam stability using these nanoparticles, the results showed that the foam volume and liquid volume in the foam was enhanced when using a smaller size and lower density of the silica nanoparticles compared with that of surfactant only. 


\section{EXPERIMENTAL}

Synthesis of the mesoporous silica nanoparticles: To synthesize mesoporous silica materials with various morphologies, a mixture solution consisting of cetyltrimethylammonium bromide (CTABr) (0.08-0.24 g), $\mathrm{NH}_{4} \mathrm{OH}$ (28 wt. \%, 0.25-1.00 $\mathrm{mL})$ and deionized $\mathrm{H}_{2} \mathrm{O}(30 \mathrm{~mL})$ was vigorously stirred for 15 min until reaching a clear solution. Tetraethylorthosilicate (TEOS) $(98 \%, 0.43 \mathrm{~mL})$ was then added into the above mixture solution and stirred for $1 \mathrm{~h}$. The reaction mixture was then left at $343 \mathrm{~K}$ overnight. From the resultant suspension solution, as-synthesized samples were retrieved through centrifugation and dried at $343 \mathrm{~K}$ overnight. The as-synthesized samples were calcined at $823 \mathrm{~K}$ for $5 \mathrm{~h}$ under an air atmosphere to remove any organic surfactant molecules.

Preparation of the decontamination foam: An alkyl polyglucoside (Elotant ${ }^{\mathrm{TM}}$ Milcoside 100; EM 100) supplied by LG Household \& Health Care in the Republic of Korea, which contains 8-10 alkyl chains as a non-ionic surfactant was used without further purification. All solutions were prepared using deionized water obtained from a Milli-Q water system. The EM 100 surfactant solution (1.0 wt. \%) was adjusted to $\mathrm{pH} 2$ by using $1 \mathrm{M} \mathrm{HNO}_{3}$. In the preparation of the decontamination foam, $0.5 \mathrm{~g}$ of the mesoporous silica nanoparticles was added into the reaction bottle, containing $50 \mathrm{~mL}$ of the EM 100 surfactant solution (1.0 wt. \%, pH 2) and stirred for several hours $(750 \mathrm{rpm})$.

Investigation of the foam stability: The foam stability was determined using a Foamscan instrument (Teclis/IT Concept, Logessaigne, France). The foam is generated by blowing nitrogen gas at a flow rate of $60 \mathrm{~mL} / \mathrm{min}$ through a porous glass filter at the bottom of a glass tube where $30 \mathrm{~mL}$ of the foaming aqueous solution at ambient temperature is placed. The foam was allowed to reach a volume of $180 \mathrm{~mL}$. The bubbling was then stopped and the evolution of the foam was analyzed using conductivity and optical measurements.

\section{RESULTS AND DISCUSSION}

The sizes, shapes and pore structures of the mesoporous silica nanoparticles were clearly revealed through the TEM and SEM analysis. Fig. 1 shows the TEM and SEM images of the mesoporous silica nanoparticles synthesized by varying the amount of the ammonia solution. It was found that the particle size and shape were strongly dependent on the amount of the ammonia solution. The pore structures of the resulting materials exhibited the existence of an ordered hexagonal array from the result of the TEM analysis except the case of using $0.25 \mathrm{~mL}$ of the ammonia solution. With the increase of the amount of the ammonia solution, the particle sizes of the mesoporous silicas increased and morphologies changed to a spherical (Fig. 1a) and rod-like (Fig. 1b-d).

The XRD patterns of the resulting materials are shown in Fig. 1(e). It is seen that mesoporous silica materials have typically hexagonal structure as a MCM-41, which can be indexed as (100), (110) and (200) in the case of using the amount of the ammonia solution over $0.4 \mathrm{~mL}$. As the amount of the ammonia solution increases, the peak intensity of the X-ray diffraction patterns scaled up suggesting a long-range order in these
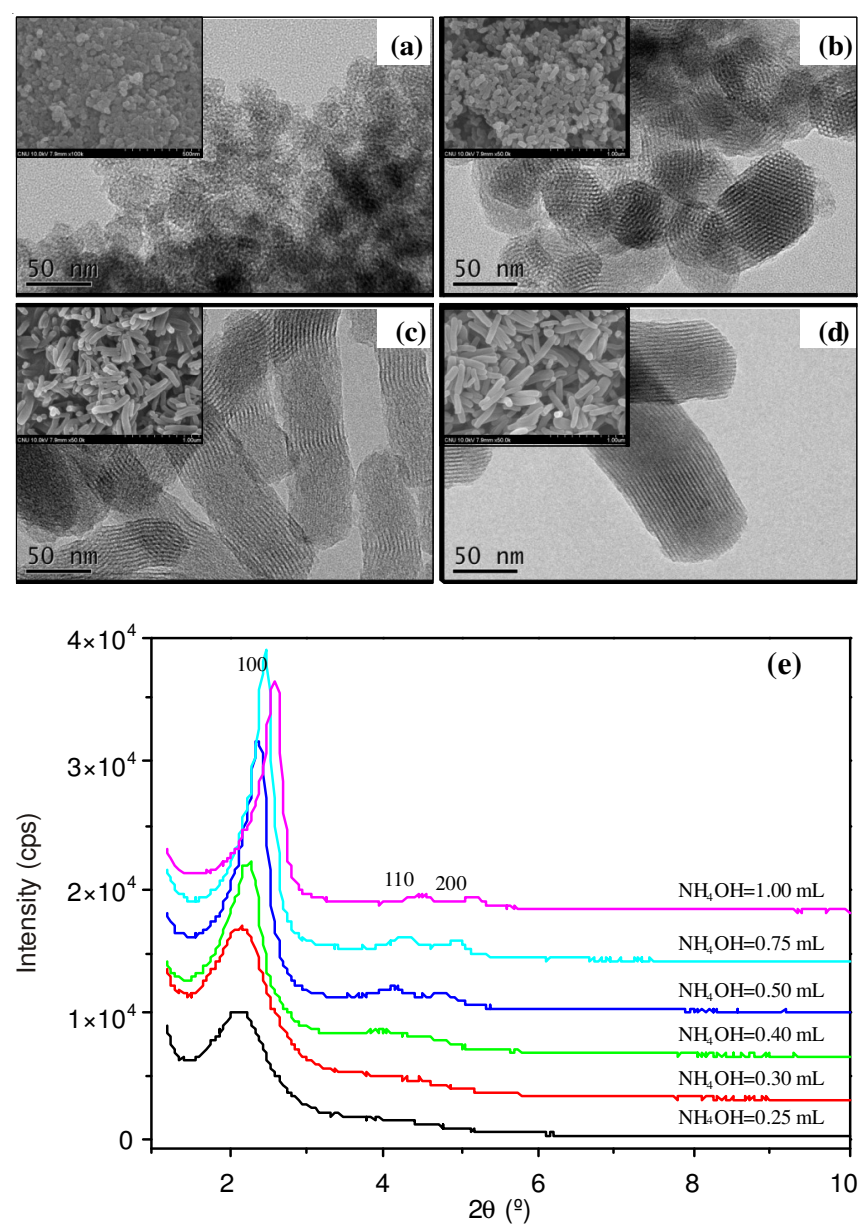

Fig. 1. Representative TEM and SEM (inset) images and X-ray diffraction patterns of the mesoporous silica nanoparticles. The amount of $\mathrm{NH}_{4} \mathrm{OH} / \mathrm{CTABr}$ : (a) $0.25 \mathrm{~mL} / 0.24 \mathrm{~g}$, (b) $0.5 \mathrm{~mL} / 0.24 \mathrm{~g}$, (c) 0.75 $\mathrm{mL} / 0.24 \mathrm{~g}$ and (d) $1 \mathrm{~mL} / 0.24 \mathrm{~g}$

materials and also slightly shifted to higher $2 \theta$ values owing to the highly condensation of the silica framework during the synthesis under stronger basic conditions.

$\mathrm{N}_{2}$ sorption isotherms at $77 \mathrm{~K}$ and their corresponding pore size distribution curves were measured for all the samples synthesized in this work and shown in Fig. 2. All the samples exhibited a type IV isotherm characteristic of mesoporous materials according to the IUPAC nomenclature due to the presence of the uniform mesopores. A steep increase of the nitrogen uptake at $\mathrm{P} / \mathrm{P}_{0}=0.15-0.3$ and $0.85-1.0$ was due to capillary condensation inside the mesopores and textured pores formed by aggregation of nanoparticles. All the samples exhibited high specific surface areas $\left(813-919 \mathrm{~m}^{2} / \mathrm{g}\right)$, large pore volumes $\left(0.60-1.66 \mathrm{~cm}^{3} / \mathrm{g}\right)$ and uniform pore sizes $(1.87-2.36$ $\mathrm{nm})$, respectively.

To investigate the influence of the particle size in the foam stability of the decontamination foam, the stability test was performed by using the mesoporous silica nanoparticles with different sizes as shown in Fig. 3. In case of using the low concentration of the CTABr, the resulting materials showed the spherical shapes, indicating the hexagonal pore structure. Fig. 4 shows the foam volume and liquid volume in the foam of the decontamination foam. From the results of the foam stability test, both foam volume and liquid volume in the foam with the addition of the mesoporous silica nanoparticles were 

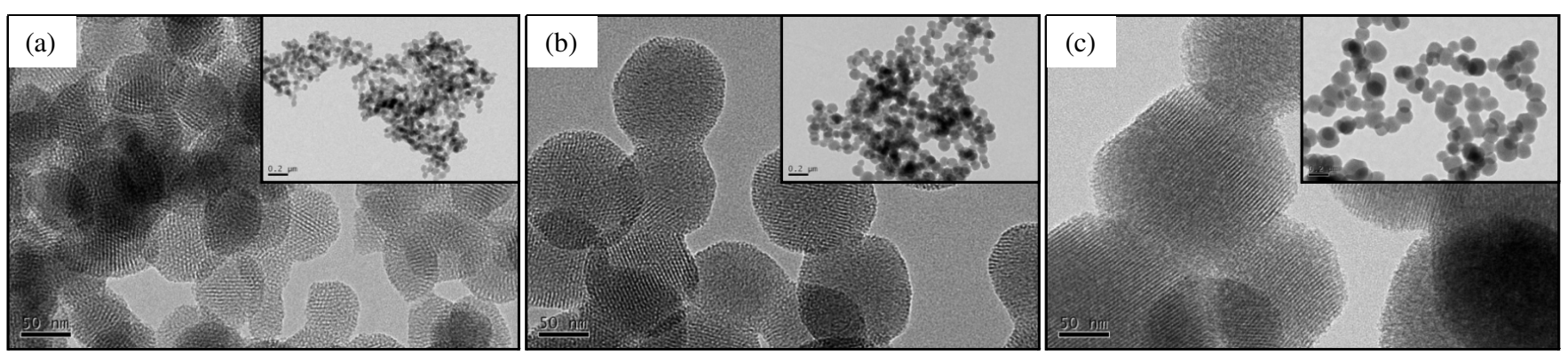

Fig. 3. TEM images of the mesoporous silica nanoparticles synthesized to investigate the foam stability. The amount of $\mathrm{NH}_{4} \mathrm{OH} / \mathrm{CTABr}$ : (a) $0.25 \mathrm{~mL} / 0.08 \mathrm{~g}$ (MS-1), (b) $0.5 \mathrm{~mL} / 0.08 \mathrm{~g}$ (MS-2) and (c) $0.75 \mathrm{~mL} / 0.08 \mathrm{~g}$ (MS-3)
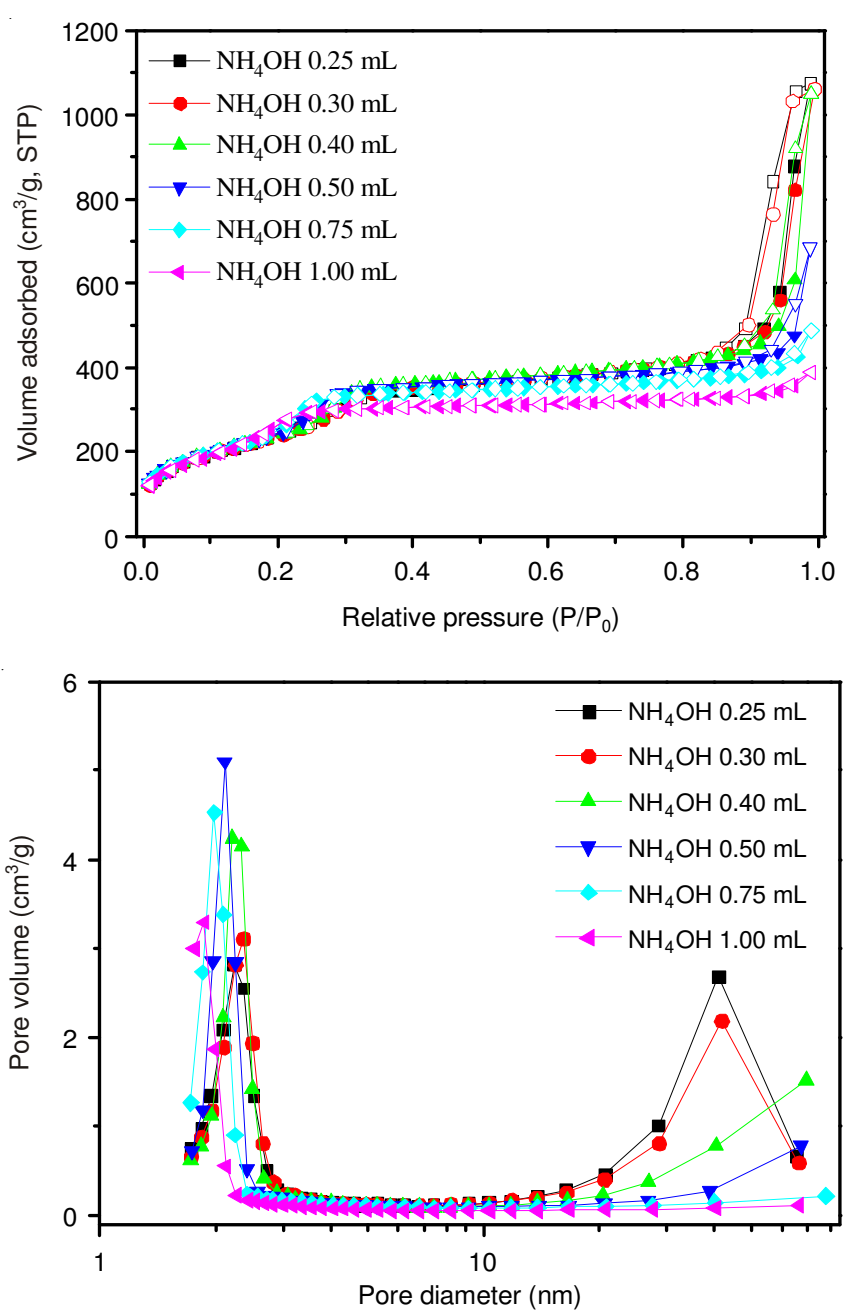

Fig. 2. $\mathrm{N}_{2}$ sorption isotherms (above) and pore size distribution curves (below) of the mesoporous silica nanoparticles

higher than those with EM 100 only. Also, the use of a smaller size showed an enhancement of the foam stability such as the foam volume and liquid volume in the foam. It was found that the foam stability was strongly dependent on the particle size.

\section{Conclusion}

Mesoporous silica nanoparticles with various sizes and shapes were synthesized by varying the amount of the ammonia solution and CTABr. As the increase of the amount of the ammonia solution, the particle sizes of the resulting materials increased and morphologies were replaced by a spherical and rod-like shape. Foam stability of the decontamination foam
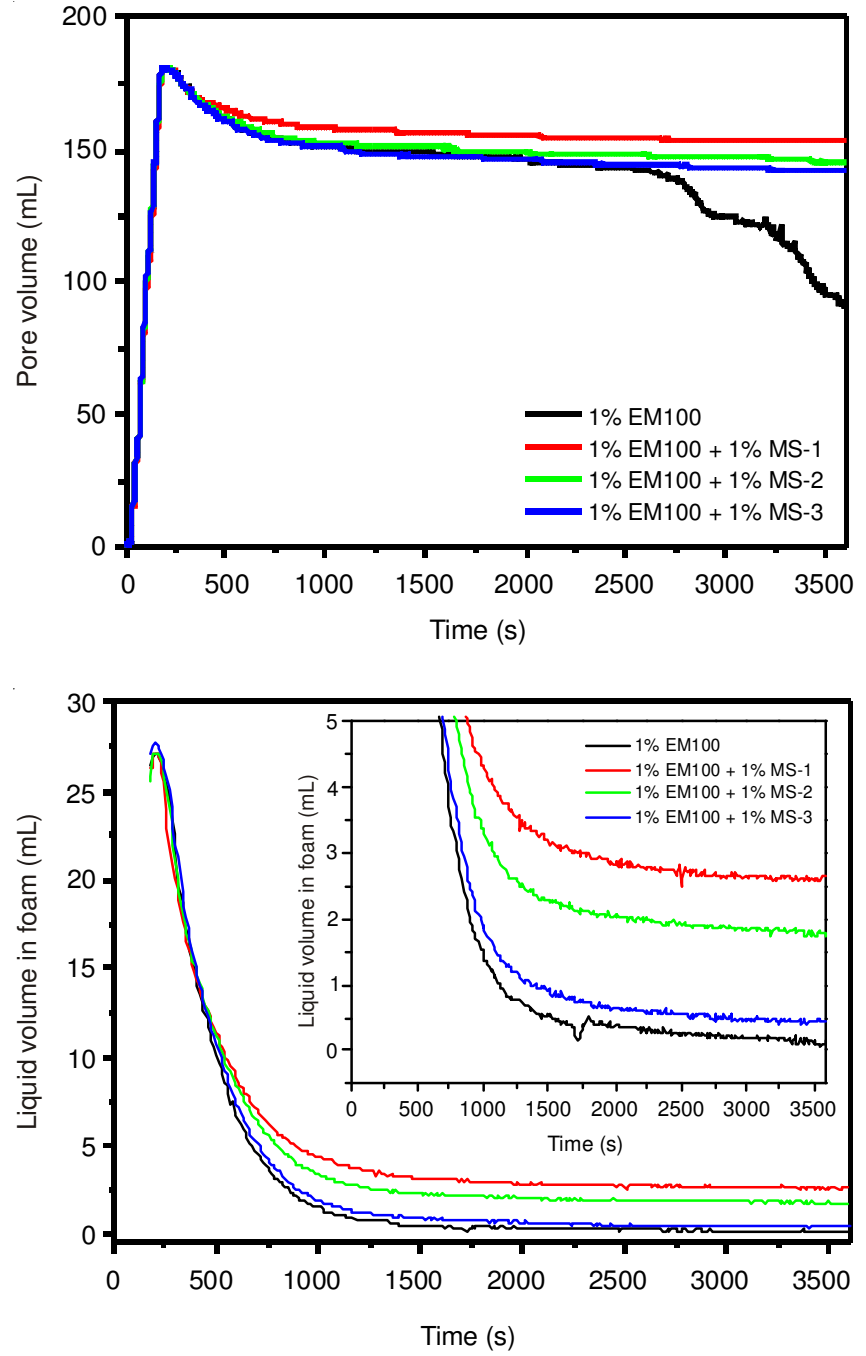

Fig. 4. Foam volume (above) and liquid volume in the foam (below) of the decontamination foam prepared by using 1.0\% EM 100, $1.0 \%$ $\mathrm{EM} 100+1.0 \% \mathrm{MS}-1,1.0 \% \mathrm{EM} 100+1.0 \% \mathrm{MS}-2$ and $1.0 \%$ $\mathrm{EM} 100+1.0 \% \mathrm{MS}-3($ at $\mathrm{pH}=2)$

prepared by using the mesoporous silica nanoparticles was higher than that with EM 100 only. The use of a smaller size showed an enhancement of the foam stability such as the foam volume and liquid volume in the foam. Further research on the foam stability of the decontamination foam prepared by using the various nanoparticles with sizes, shapes, structures and surface properties of the nanoparticles is currently underway. 


\section{ACKNOWLEDGEMENTS}

This work has been carried out under the Nuclear R\&D Program (2012M2A8A5025655) funded by Ministry of Science, ICT \& Future Planning.

\section{REFERENCES}

1. C.T. Kresge, M.E. Leonowicz, W.J. Roth, J.C. Vartuli and J.S. Beck, Nature, 359, 710 (1992).

2. S.A. Bagshaw, E. Prouzet and T.J. Pinnavaia, Science, 269, 1242 (1995).

3. Q.S. Huo, R. Leon, P.M. Petroff and G.D. Stucky, Science, 268, 1324 (1995).

4. Q. Lu, F. Gao, S. Komarneni and T.E. Mallouk, J. Am. Chem. Soc., 126, 8650 (2004).

5. X. Pang, J. Gao and F. Tang, J. Non-Cryst. Solids, 351, 1705 (2005).

6. S. Kang, Y.B. Chae and J.-S. Yu, J. Nanosci. Nanotechnol., 9, 527 (2009).
7. Q. Cai, Z.-S. Luo, W.-Q. Pang, Y.-W. Fan, X.-H. Chen and F.-Z. Cui, Chem. Mater., 13, 258 (2001).

8. M. Grün, K.K. Unger, A. Matsumoto and K. Tsutsumi, Micropor. Mesopor. Mater., 27, 207 (1999).

9. K. Schumacher, M. Grün and K.K. Unger, Micropor. Mesopor. Mater., 27, 201 (1999).

10. M.T. Bore, S.B. Rathod, T.L. Ward and A.K. Datye, Langmuir, 19, 256 (2003).

11. S.B. Yoon, J.-Y. Kim, J.H. Kim, Y.J. Park, K.R. Yoon, S.-K. Park and J.-S. Yu, J. Mater. Chem., 17, 1758 (2007).

12. S.B. Yoon, C.-K. Kim, D.-H. Jung, B.-S. Choi, W.-K. Choi, K.-W. Lee and J.-K. Moon, Asian J. Chem., 25, 7023 (2013).

13. S. Faure, P. Fuentes and Y. Lallot, US Patent, US 2008/0228022 A1 (2008).

14. S. Guignot and S. Faure, US Patent, US 2010/0069281 A1, (2010). 